\section{Chances and challenges of comprehensive geriatric assessment training for healthcare providers}

\author{
Maria Cristina Polidori, ${ }^{1}$ \\ Regina Elisabeth Roller-Wirnsberger ${ }^{2}$ \\ ${ }^{1}$ Ageing Clinical Research, Department \\ of Internal Medicine II, University \\ Hospital of Cologne, Germany; \\ ${ }^{2}$ Department of Internal Medicine, \\ Medical University of Graz, Austria
}

\begin{abstract}
Geriatrics is the medical specialty that deals with physical, psychological, social and functional aspects of the older adult and represents a most authentic form of personalized medicine. As such, it is successfully performed through its cornerstone instrument, the comprehensive geriatric assessment (CGA), which consistently shows its significant benefits since over 30 years. However, the high speed at which the world population ages and gets affected by chronic conditions, multimorbidity and functional impairment is not yet accompanied by a strong, competent and self-conscious geriatric culture. This unmet need contributes to the further exponential increase of disability and lack of well-being in older persons. In this paper, we give an overview of the possible reasons for the lack of adequate CGA implementation across settings and highlight the likely benefits of consequent training and education of two groups of (future) professionals in the field of aging medicine: medical students and residents on one hand and multiprofessional healthcare providers, particularly nurses and physiotherapists, on the other hand.
\end{abstract}

\section{Introduction}

In our aging societies, an increasing deal of attention is being shifted towards improvement of care for older adults across settings by on one hand implementing existing tools which have been shown to be effective against poor outcomes and on the other hand by developing new strategies to optimize healthcare interventions. The comprehensive geriatric assessment (CGA), evidently the cornerstone of geriatric medicine, is known to produce considerable amelioration in the quality of care provided to older adults, as assessed by critical healthcare quality indicators including discharge home, rehospitalization rate, and mortality. These meso-indicators have of course immediate impact at the macro-indicator level of socioeconomy and public health. Most importantly, however, the correct use of the CGA has been shown to improve substantially the fundamental micro care level of personal dignity, quality of life, mood and wellbeing of the older patient.

This success bases upon the fundamental premise of the multidimensionality of the person; the fact that the physical problem the older patient presents with is most frequently co-caused by a multitude of organ-unrelated factors including psychosocial and functional ones; and as a consequence, the fact that intervening on a presumed organ failure (or sometimes even worse, intervening on a biomarker to correct it) will be even in the presence of temporary success a mere academic exercise.

The crucial scientific achievement - the proof that CGA dramatically improves outcomes and life trajectories of older patients and therefore their caregivers - has been consistently shown among thousands of community dwellers, hospitalized persons, General Practitioner (GP) utilizers and Emergency Department (ED) visitors. ${ }^{1,2}$ However, the establishment and routine use of the CGA or special care units within EDs, GPs and hospitals where older patients are preferentially hosted and multidimensionally assessed is not achieved yet. In other words, there is a gap between what is known and what is being performed in the clinical care of the older person.

While the detailed description of CGA studies goes beyond the scope of this paper, the reasons for poor CGA implementation, already described in detail elsewhere, ${ }^{3}$ will be recalled below. Based on the information of a $15 \%$ rehospitalization rate at one month only for older ED guests, ${ }^{4}$ as well as on the current average 17 years lived with disability pro person, or the up to $50 \%$ overall population passing away outside their homes (Dpt Health London), ${ }^{5}$ the motto among geriatricians better care is always cheaper at the end counteracts the critique that the CGA might be expensive to perform.

In this optic, the aim of the present article is to present the importance of fostering geriatric education in order that healthcare professionals do expect steadily older patients and are equipped with appropriate knowledge when treating them. Indeed, it is important that students and healthcare providers for older patients grasp the theory of CGA, which reflects the multidimensionality of the person. Trainees must be made aware that even the most advanced therapy
Correspondence: Maria Cristina Polidori, Ageing Clinical Research, Department II Internal Medicine, University Hospital of Cologne, Germany.

E-mail: maria.cristina-polidori@uk-koeln.de

Key words: Minimum geriatric competencies; comprehensive geriatric assessment; education; geriatric team; undergraduate; postgraduate; healthcare professionals.

Received for publication: 2 October 2018. Accepted for publication: 25 October 2018.

This work is licensed under a Creative Commons Attribution-NonCommercial 4.0 International License (CC BY-NC 4.0).

(C) Copyright M.C. Polidori and R.E RollerWirnsberger, 2018

Licensee PAGEPress, Italy

Geriatric Care 2018; 4:7853

doi:10.4081/gc. 2018.7853

applied in the best way and with the most appropriate indication will be subjected, in advanced age, to a high risk of failing or in fact of being counterproductive if risk or presence of geriatric syndromes or psychosocial and functional needs are not assessed in an interactive way. To achieve its aim, the present work will focus on the factors inhibiting the appropriate CGA performance, followed by the proposal that an appropriate CGA teaching is not only essential in undergraduate and postgraduate medicine, but also across medical disciplines and non-medical professions taking part in the team assessment and management of older patients.

\section{The know-do gap in the implementation of comprehensive geriatric assessment}

In a brilliant overview published in Age\&Ageing in 2016, Gladman and collegues listed a number of factors possibly impeding the correct and consequent implementation of the CGA across geriatric settings. ${ }^{3}$ The performance of the CGA requires important competencies and financial resources to be performed routinely in community-dweller settings with preventive purposes. Although it may seem extremely difficult to improve CGA-related skills among health practitioners and financially cover enough of them to perform the CGA routinely across settings, the societal costs of hospitalization and re-hospitalization rates as well as nursing home admissions are not sustainable. Most importantly, 
the consequences of an inadequate implementation of a comprehensive evaluation of older multimorbid patients and not compatible with a dignified quality of life of this patient population.

Marjorie Warren, a surgeon, observed, 1935, that the hospitalized chronic ill is rather to be classified according to the degree of impairment hindering his functioning (immobility and cognitive impairment) than following the reason of admission and treated organ. Among the several hospital processes, she positively influenced towards older personoriented medicine. Warren advocated teaching medical students about the care of older people by senior doctors with special interest in geriatrics. ${ }^{6}$ This prompted several initiatives, several of which nurse-based, for the improvement of geriatric care, but the first structured tool to perform patient-centered diagnosis and treatment was described several decades later, by Lawrence Rubenstein. ${ }^{7}$ The four dimensions of the older patient as a person must, per definition, be accurately evaluated as their adequacy will profoundly impact the success of any medical intervention planned and performed.

Three decades and a couple of hundred studies after the first description of CGA, it is known, thanks also to the recent metaanalyses of Ellis, ${ }^{1,2}$ that the implementation of the CGA as both a diagnostic and therapeutic tool leads to improvement of diagnosis, function, discharge destination, mood, behavior and cognition while reducing polypharmacy, mortality, nursing home admission, hospitalization rates and therefore costs. Nonetheless, a huge amount of healthcare settings including many hospitals do not offer this opportunity to older patients: a paradox in light of the fact that vulnerable patient populations are the mostly admitted to acute care hospitals and practices, thereby at highest risk of poor outcomes.

Gladman et al. ${ }^{3}$ discussed a number of barriers to the implementation of CGA, including guidelines factors, professional factors, patient factors, professional interactions, incentives, resources, capacity for organizational change, as well as social, political and legal factors. While professionals in the field of aging medicine agree on all these still highly current obstacles, there is little sense in not actively working on the suggested method to overcome these barriers, co-production of research, practice communities and knowledge brokers. ${ }^{3}$ These three aspects, reflected in the three main pillars of academic medicine - research, clinical activity and teaching - might be performed also in concerted actions of independent players and might be enormously fostered by focusing on education and training, not only on the medical level (medical students and trainees) but also on the healthcare provider side (geriatric team multiprofessional representatives).

\section{Comprehensive geriatric assessment education and training}

With the rise in the number of older and vulnerable adults, there is an urgent need not only of doctors skilled in Geriatric Medicine, ${ }^{8,9}$ but of as many doctors and healthcare providers as possible competent in the complex management of older patients. Medical students, residents in Geriatrics or in other disciplines, nurses and therapists, nutritionists, spiritual care providers, psychologists and any specialist involved in the multidisciplinary geriatric team, need to be prepared about the significance of (re)acting in an age-attuned way, and expecting older patients - in the ED, as inpatients, in the outpatient clinic, in longterm care facilities like in any other geriatric setting. ${ }^{10}$

The best proof of the public health and public policy recognition that older multimorbid patients have to be most promptly triaged to geriatric interventions is the fact that, in recent years, screening instruments have been developed and evaluated in their efficacy to identify directly on admission geriatric patients at risk of frailty or adverse outcomes or those benefiting from rehabilitative interventions. Their efficacy, however, has been shown to be limited, ${ }^{11,12}$ because personalized medicine cannot be rapidly conducted with yes/no questions. Older patients in settings where typically only short time is available (such as the ED or the GP outpatient clinic) are often alone, may consider their complaints as normal signs of being older, might be acutely disorientated, and/or sensorially impaired, etc. Communication alone and history collection can be extremely difficult in ageing medicine, which is the reason why, as mentioned below, both are highlighted outcomes of the undergraduate curriculum in geriatric medicine ${ }^{8} \quad$ (http://uemsgeriatricmedicine.org/www/dok/Minimum\%20 Training\%20Requirment\%202016.pdf). These soft skills, together with minimum geriatric competencies for undergraduate medical students strongly impact the quality of care for older adults, and educational experience collected so far with respect to medical students might serve as basis of a structured training for non-physicians multiprofessionals. In this light, the term geriatric education onwards will be used to intend both medical and non-medical geriatric education.

One of the greatest challenges to goaloriented geriatric education is that many learners today are trained in Geriatric Medicine at remote clinical sites, i.e. away from the university campus. This is highly valid for non-medical disciplines. Therefore, prompt reaction from the teacher's side to students' training deficits is challenging; curriculum directors in the field face the demands associated with this issue. It is well known, in fact, that the capability of faculty members as role models has an impact on the success and sustainability of continous educational programs, teaching content and professionalism. ${ }^{13,14}$ In addition, a role model faculty member is effective in transferring a kind of hidden curriculum which typically includes attitudes, activities and teaching behaviours beyond the formal curriculum. This implies that teachers are needed who not only fulfill the formal requirements of being practicing specialists in Geriatric Medicine for at least 5 years, but who are equipped with solid didactic knowledge and personality skills. Awareness of and ability in teaching methods are fundamental instruments to deliver the complex learning objectives and professional behaviors developed by the Geriatrics Societies (http://uemsgeriatricmedicine.org/www/dok/Minimum $\% 20$ Training\%20Requirment\%202016.pdf). In this context, distant teaching using blended learning including workshops, small-group case discussion, online video, and an online discussions of geriatric cases have been demonstrated to be effective and efficient ${ }^{15}$ and may also be a resource for further improvement and development of training in Geriatric Medicine.

From this perspective, it is not surprising that only a low number of medical faculties across Europe provide teaching to cover topics of the complex care management of older people $e^{8,16,17}$ and that, at a European level, geriatric education is extremely heterogeneous. ${ }^{18}$ To foster undergraduate education in Geriatrics, the American as well as European associations and societies of Geriatric Medicine have agreed very recently upon minimum geriatric competencies in undergraduate medical training which are considered critical for the adequate medical management of the older adult. ${ }^{8,19}$ Although the training for MD students and non-MD trainees is quite heterogeneous across different institutions, there are several progresses which have been made in the past recent years through the production of the catalogues cited above. As these are critical for an optimal clinical performance with older patients, 
and are required skills for the performance and interpretation of a CGA, healthcare providers other than medical students and residents should be regularly exposed to this structured kind, even if in a modified way, of educational curriculum.

Performance, application and interpretation of CGA along with planning complex interventions are major teaching outcomes for medical students and colleagues at the post-graduate level. Within this frame, outcome-based education advocated for design and delivery of a health professional curriculum of the $21^{\text {st }}$ century shall be consistently and homogenously applied also to non-medical multiprofessionals constituting the geriatric team. Outcome-based education implies that professional competencies required for future practice are defined in terms of learning outcomes. These include not only knowledge - like in traditional education - but also skills and attitudes which are commonly understood as soft abilities but in real life might in fact decisively influence trajectories and outcomes especially of older adults. Following this construct, learning objectives, therefore, are formulated from and aligned to the end-product capability identified. A fundamental step of outcome-based education includes the fact that assessment tools of learners are designed ad hoc to evaluate the desired attribute of the end-product. Pragmatically, the learner of geriatric outcome-based education - whether MD student or non-medical healthcare professional such as a physiotherapist, a nurse, or a nutritionist - should be assessed in his/her skills, after a period of learning under supervision, by means of his/her ability to independently perform a given set of professional tasks. These professional tasks are called entrustable professional activities (EPAs) and include, in the case of CGA, the independent performance of the individual domains of the CGA itself.

While the recently published competencies to be achieved in Geriatric Medicine by medical undergraduates are strongly built upon Benner's developmental model of a learner ${ }^{20}$ and grounded on the Bloom's cognitive taxonomy, ${ }^{21}$ the evaluation outcomeoriented EPAs approach is intellectually linked to the well-established concept that the identification of training needs paves the path to the design, preparation and delivery of training; the latter leads to reaction to and learning from the training, which will be followed by the transferal of training to the workplace where finally the impact of the training on the organization will be measured; this will in turn prompt further identification of training needs and the positive feedback circle will ideally continue. ${ }^{22}$

This approach may help to raise accept- ance by students of the training content as they see the direct connection with the clinical care pathways. As medical students and non-medical professionals are adult learners, they need to have a concrete experience of knowledge content applied either in simulation scenes or with patients, upon which they can than reflect either in a group or led by a tutor. Through their reflection they are able to formulate abstract concepts and may test the implications of their knowledge in individual situations. With respect to CGA, by giving trainees the opportunity to perform this diagnostic and therapeutic approach in a real-life situation, a learning environment will be provided in which they can reach the highest competence level according to the Miller's pyramid.

All-in-all, this kind of feed-back and feed-foward from teaching to practice and back is called constructive/operative alignment and bases upon the concept that of course becoming a professional is a social process that in large part needs to take place in a context where the profession is practiced. Within this learning process, if the constructive aligment is adequate, members (learners and teachers) develop and maintain a joint enterprise, mutual engagement and a shared repertoire. Unfortunately, constructed aligment is not obvious and there is a strong need worldwide in medicine in general and especially in geriatrics of systematically adapting in a bidirectional way theoretical costructs to practice.

A huge challenge to the presented outcome- and objective-oriented learning is the currently running knowledge-based and classroom-oriented medical program. Therefore, a strong paradigm shift in teaching activities is necessary, which is under a great deal of attention ${ }^{23-26}$ and might well include, in a modified way, the geriatric multiprofessional team.

Careful planning of an integrated and step-wise educational approach exposing learners repetitively during their training to geriatric content is mandatory for the implementation of successful and sustainable curricula for medical management of older patients with complex care needs. Whatever teaching method a faculty chooses to train students, residents and healthcare providers in the performance and interpretation of CGA, a detailed program evaluation should follow. Using models of evaluation such as the one by Spilsbury and colleagues ${ }^{22}$ evidence on training in the field of Geriatrics may be provided and bids for resources from training bodies may be supported.

Planning educational events and curricula for geriatric education raises the question what is the basic competence to be achieved for CGA. Basic skills should become a common platform to be shared also with professional figures from different disciplines. As outlined in the recommendations published by Masud and collaborators Graduates should have the special skills needed to conduct a history and perform an assessment in an older patient, ${ }^{8}$ this fundamental approach might be applied to the geriatric team within each specialty. Indeed, this learning objective clearly outlines that students need to acquire basic skills to handle an assessment situation for various functional domains, outlined in more detail within the recommendation for the minimum geriatric competences. This implies that giving access to information on functionality in old age and the CGA as an instrument to assess this functionality is the first step in the educational helix. As outlined earlier, this information may be offered either through live classroom educational events or using blended learning formats involving information communication technologies (ICT) - learning modules together with live educational events (Table 1). There is currently some evidence that E-learning-based medical education can be beneficial for learners as well as educators. Web-based learning may improve students' and trainees' adherence and compliance as it offers the opportunity to „consume“ learning content whenever convenient and for the learners to have as many repetitions as they perceive that they need. Furthermore, outcome assessment can be determined directly within the e-learning module. ${ }^{27}$ However, information as a stand-alone offer will not be sufficient to drive undergraduate medical students towards the competence level requested within the European recommendations. The most appropriate educational format for CGA is clinic or bedside teaching, especially if performed with the interdisciplinary geriatric team with its multiprofessional membership..$^{28}$ At this level and at a subsequent management one, students and trainees will learn the importance of being members of a flat-hierarchy interdisciplinary team with multiprofessionals for nonmedical disciplines, where each professional performs within the frame of a comprehensive assessment, with each single domain loosing significance if extrapolated from the personalized multidimensional context.

Team-playing in co-management with other expertise has been shown to enable more effective implementation of inter-professional learning activities and assessment within the core curriculum. ${ }^{28}$ Special attention should be given to the role and structure of the geriatric team. In fact, in every developmental step of a preliminary management plan for patients presenting with 
Table 1. Teaching and evaluation formats for comprehensive geriatric assessment.

Teaching formats

Knowledge on CGA

\begin{tabular}{ll} 
Technical skills & $\begin{array}{l}\text { Simulation patients, E-learning modules, bedside } \\
\text { teaching }\end{array}$ \\
\hline Communication skills during CGA & $\begin{array}{l}\text { Simulation training, bedside teaching using various } \\
\text { educational approaches }\end{array}$
\end{tabular}

Interpretation of results and care planning/professionalism

CGA, comprehensive geriatric assessment.
Classroom teaching, self-directed learning of given content, E-learning modules, webinars
(Simulation training), bedside teaching

CCA, comprehensive geriatric assessment.
Evaluation formats

Multiple choice in different formats, short answer assessment, short essay assessment, structured oral assessments

OSCE (Objective structured clinical examination), PACES (Practical Assessment of Clinical Examination) Skills, Mini-CEX (Mini Clinical examination assessment)

OSCE (Objective structured clinical examination), PACES (Practical Assessment of Clinical Examination Skills, Mini-CEX (Mini Clinical examination assessment) Mini-CEX, DOPS, $360^{\circ}$ assessment, Case-based Discussion, Clinical \& Educational Supervisor Reports functional deficits, including adaptive interventions, the involvement of interdisciplinary team members from appropriate disciplines, such as social work, nursing, rehabilitation, nutrition, and pharmacy needs to be clearly described and highlighted. The educational formats of simulation training and bedside teaching after theoretical lectures, case scenarios and educational videos fit perfectly for the teaching of CGA, especially if the activity of the geriatric team can be demonstrated.

While exposing the basis of Geriatrics, the essential nature of the discipline, which is multidimensional, crossing and going beyond the organ approach is presented by the teacher. For the geriatric team, the meaning of multidimensionality, obvious to geriatrics experts, can be deepened and guided beyond the challenges of struggling with time-pressure medicine. The unavoidably personalized character of geriatric medicine and management faces evidencebased medicine and its challenges in advanced age and the fundamental gerontological concept of the heterogeneity of ageing. By rendering the comprehensive yet individual strategies as key points for a successful treatment and rehabilitation plan, an understanding of CGA as representing personalized medicine will be facilitated.

No personalized medicine can be conducted without soft skills. So-called soft skills like appropriate communication with older persons including those with cognitive or sensory impairment are included in the frame of a main geriatric curriculum outcome, i.e. graduates should respect patients regardless of their age. ${ }^{8,26,29}$ Within the latter, an important professional behaviour to be conveyed is the maintenance of a personcentered and professional approach to the older person; this skill, as well as empathy beyond the dichotomy between biomedicine and the humanities, ${ }^{30}$ has important ethical implications and is to be encouraged within the hard core of geriatric medicine. At an undergraduate level, to demonstrate empathy and improve the technical skills described below, simulation training is being successfully adopted where students are exposed to the several impairments occurring with increasing age. ${ }^{31}$

Finally, and as discussed above, the assessment of learning success seems to play a major role in driving students' as well as residents' learning behavior. As informed by the Miller's pyramid, assessment formats have to be strictly aligned with the competence levels of outlined learning outcomes. This means that during conceptualization of the curriculum for teaching CGA, colleagues should consider how the learning outcomes of trainees are to be assessed. Table 1 outlines possible training and assessment formats already used by many faculties across the globe, which might be adapted for regular updates to multiprofessionals. The decisions on how to teach and evaluate CGA strongly depends on the needs of trainees and given contextual factors. The number of students to be trained, the availability of teaching staff and time as well as the structural environments strongly influence the didactic decisions in curriculum development.

In conclusion, teaching CGA is a demanding task, which encompasses several levels of difficulty including imparting the importance of communication along with technical skills. Once the understanding of the latter is ensured, the multidimensionality has to be explained. Beyond the use of scales, the complexity of the patient and interpretation of CGA results should be clear to the medical students, trainees and multidisciplinary professionals. Finally, using CGA to implement plans of care in different settings is the competence to be achieved at novice and experienced resident and postgraduate level. This latter phase, in addition to improvement through gaining experience, might be further deepened by conveying the use of CGA tools including apps to assess multidimensional prognosis and thereby improving clinical decision making in older patients. ${ }^{32-35}$ Indeed, it has been recently shown that targeted educational events using operatively aligned outcome-centered learning objectives improves the effects of geriatric multidimensional interventions on patient overall outcomes as assessed by the multidimensional prognostic index (MPI). In these preliminary observations, ${ }^{36-38}$ a team specifically trained based upon constructive alignment of theoretic learning objectives with practical learning outcomes, produces more significant improvements in the CGAbased MPI - already shown to accurately measure overall in-hospital disease/recovery course $^{39}$ - from admission to discharge with respect to standard of care. In the latter, the multiprofessional members of the geriatric team perform their individual tasks individually and discuss the results weekly. This suggests that delivery of learning objectives in the frame of targeted outcome-oriented education may substantially improve the efficacy of the concerted geriatric team action as evaluated by an objective outcome measure such as the MPI. More research is needed on this topic before guidelines are developed.

\section{References}

1. Ellis G, Whitehead MA, Robinson D, et al. Comprehensive geriatric assessment for older adults admitted to hospital: meta-analysis of randomised controlled trials. BMJ 2011;27:343:d6553.

2. Ellis G, Gardner M, Tsiachristas A, et al. Comprehensive geriatric assessment for older adults admitted to hospital. Cochrane Database Syst Rev 2017;9: CD006211. 
3. Gladman JR, Conroy SP, Ranhoff AH, Gordon AL. New horizons in the implementation and research of comprehensive geriatric assessment: knowing, doing and the 'know-do' gap. Age Ageing 2016;45:194-200.

4. Biese KJ, Busby-Whitehead J, Cai J, et al. Telephone follow-up for older adults discharged to home from the Emergency Department: a pragmatic randomized controlled trial. J Am Geriatr Soc 2018;66:452-8.

5. Department of Health. End of Life Care Strategy - promoting high quality care for all adults at the end of life. London: Department of Health; 2008.

6. Warren M. The evolution of a geriatric unit from a public assistance institution, 1935-1947. Proc R Soc Med 1948; 41:337.

7. Rubenstein LZ. Specialized geriatric assessment units and their clinical implications. West J Med 1981;135: 497-502.

8. Masud T, Blundell A, Gordon AL, et al. European undergraduate curriculum in geriatric medicine developed using an international modified Delphi technique. Age Ageing 2014; 43:695-702.

9. Hafner K. As population ages, where are the geriatricians? The New York Times, January 2016. Available from: https:// www.nytimes.com/2016/01/26/health/w here-are-the-geriatricians.html? $\mathrm{r}=0$

10. Blundell A, Masud T. Teaching and learning the content of geriatric medicine. In: Roller-Wirnsberger RE, Singler K, eds. Learning Geriatric Medicine. Berlin: Springer; 2018.

11. Carpenter CR, Shelton E, Fowler S, et al. Risk factors and screening instruments to predict adverse outcomes for undifferentiated older emergency department patients: a systematic review and meta-analysis. Acad Emerg Med 2015;22:1-21.

12. Yao JL, Fang J, Lou QQ, Anderson RM. A systematic review of the identification of seniors at risk (ISAR) tool for the prediction of adverse outcome in elderly patients seen in the emergency department. Int $\mathrm{J}$ Clin Exp Med 2015;15:4778-86.

13. International Conference hosted by the German Rectors' Conference (HRK). The structure of Medical Education in Europe: Implementing Bologna - on the way to a European success story?. Berlin: German Medical Science GMS Publishing House; 2008.

14. Association for Medical Education in Europe and World Federation for
Medical Education. Statement on the Bologna Process and Medical Education; February 2005. Available from: https://www.aic.lv/ace/ace_disk/ Bologna/contrib/Statem_oth/WFMEAMEE.pdf, accessed Dec 7 th 2018

15. Goldman LN, Wiecha J, Hoffman M, Levine SA. Teaching geriatric assessment: use of a hybrid method in a family medicine clerkship. Fam Med 2008; 40:721-5.

16. Kolb GF. Survey of the German Geriatrics Society on the academic situation, student education and the state of further training. Eur J Geriatrics 2005; 7:239-48.

17. Chenot JF. Undergraduate medical education in Germany. German Med Sci GMS 2009;7:1-11.

18. Roller-Wirnsberger RE, van den Noortgate N, Bonin-Guillaume S, et al. Setting competencies and standards for a European Leadership Program in Geriatric Medicine: "The European Academy for Medicine of Ageing (EAMA) reloaded". Eur Geriatr Med 2018;9:399-406.

19. Leipzig RM, Granville L, Simpson D, et al. Keeping granny safe on July 1: a consensus on minimum geriatrics competencies for graduating medical students. Acad Med 2009;84:604-10.

20. Benner P. From Novice to expert. Boston: Addison-Wesley Publishing; 1984.

21. Bloom B. A Handbook of Educational Objectives. The Cognitive Domain. New York: McKay; 1964.

22. Spilsbury M. Measuring the Effectiveness of Training. Brighton: IES; 1995. p 12, Figure 3.1.

23. Miller GE. Assessment of clinical skills/competence/performance. Acad Med 1990;65:S63-7.

24. Windhaber T, Koula ML, Ntzani E, et al. Educational strategies to train health care professionals across the education continuum on the process of frailty prevention and frailty management: a systematic review. Aging Clin Exp Res 2018. [Epub ahead of print].

25. Roller-Wirnsberger RE, Polidori MC, Singler K. Teaching comprehensive geriatric assessment. In: Pilotto A and Martin F, eds. Comprehensive Geriatric Assessment. Berlin: Springer; 2018.

26. Bowen JL. Educational strategies to promote clinical diagnostic reasoning. N Engl J Med 2006;355:2217-25.

27. Ruiz JG, Mintzer MJ, Leipzig RM. The impact of E-Learning in Medical Education. Acad Med 2006;81:207-12.
28. O’ Keefe M, Henderson A, Chick R. Defining a set of common interprofessional learning competencies for health profession students. Med Teach 2017 [Epub ahead of print].

29. Bombeke K, Symons L, Vermeire E, et al. Patient-centredness from education to practice: The "lived" impact of communication skills training. Med Teach 2012;34:e338-48.

30. Halperin EC. Preserving the humanities in medical education. Med Teach 2010;32:76-9.

31. Braude P, Reedy G, Dasgupta D, et al. Evaluation of a simulation training programme for geriatric medicine. Age Ageing 2015;44:677-82.

32. Dent E, Kowal P, Hoogendijk EO. Frailty measurement in research and clinical practice: a review. Eur J Intern Med 2016;31:3-10.

33. Wernier RM, van Rossum E, van Velthuijsen E, et al. Validity, reliability, and feasibility of tools to identify frail older patients in inpatient hospital care: a systematic review. J Nutr Health Aging 2016;20:218-30.

34. Pilotto A, Ferrucci L, Franceschi M, et al. Development and validation of a multidimensional prognostic index for oneyear mortality from comprehensive geriatric assessment in hospitalized older patients. Rejuvenation Res 2008; 11:151-61.

35. Singler K, Roth $\mathrm{T}$, Beck $\mathrm{S}$, et al. Development and initial evaluation of a point-of-care educational app on medical topics in orthogeriatrics. Arch Orthop Trauma Surg 2016;136:65-73.

36. Polidori MC. MPI e la formazione del personale sanitario. In: Proceedings of the Annual Congress SIGOT 2017, Genova, Italy.

37. Polidori MC. Education of Healthcare professionals about the multidimensional approach to the older person. In: Proceedings of the 2017 Annual Congress of the European Geriatric Medicine Society, Nizza, France.

38. Pickert L, Meyer AM, Noetzel N, et al. The role of prognosis for tailored multidimensional interventions in older multimorbid patients: the Multidimensional Prognostic Index. Accepted for presentation at the 2018 Annual Congress of the European Geriatric Medicine Society, Berlin, Germany.

39. Volpato S, Daragjati J, Simonato M, et al. Change in the multidimensional prognostic index score during hospitalization in older patients. Rejuvenation Res 2016;19:244-51. 